\title{
CREATION PROBABILITIES OF HIERARCHICAL TREES
}

\author{
A. Olemskoi ${ }^{1,2}$, S. Borysov ${ }^{2}$, I. Shuda ${ }^{2}$ \\ ${ }^{1}$ Institute of Applied Physics, Nat. Acad. Sci. of Ukraine, \\ 58, Petropavlovskaya St., 40030, Sumy, Ukraine \\ ${ }^{2}$ Sumy State University, 2, Rimskii-Korsakov St., 40007, Sumy, Ukraine \\ (Received March 31, 2010; received in final form September 30, 2011)
}

\begin{abstract}
We consider both analytically and numerically the creation conditions of diverse hierarchical trees. A connection between the probabilities to create hierarchical levels and the probability to associate these levels into a united structure are studied. We argue that a consistent probabilistic picture requires the use of deformed algebra. Our consideration is based on the study of the main types of hierarchical trees, among which both regular and degenerate ones are studied analytically, while the creation probabilities of Fibonacci and free-scale trees are determined numerically. We find a general expression for the creation probability of an arbitrary tree and calculate the sum of terms of deformed geometrical progression that results from the consideration of the degenerate tree.
\end{abstract}

Key words: probability, hierarchic tree, deformation.

PACS number(s): 02.50.-r, 89.75.-k, 89.75.Fb

\section{INTRODUCTION}

The problem of the origin of hierarchy and its implications for physical, biological, economical, ecological, social and other complex systems has a long history which can be found in Refs. [1-13]. Along this line, one of the most striking manifestations of hierarchy is found in complex networks $[14,15]$. As is shown for diverse systems, ranging from the World Wide Web [16] to biological [17-20] and social [21-23] networks, real networks are governed by strict organizing principles displaying the following properties: (i) most networks have a high degree of clustering; (ii) many networks have been found to be scale-free $[24,25]$ that means the probability distribution over node degrees, being the set of the numbers of links with neighbours, follows a power law. Moreover, many networks are modular: one can easily identify groups of nodes that are highly interconnected with each other, but have only few or no links to nodes outside the group to which they belong (in society such modules represent groups of friends or coworkers [26], in the WWW these denote communities with shared interests [27], in the actor network they characterize specific genres or simply individual movies). This clearly identifiable modular organization is at the origin of the high clustering coefficient seen in many real networks. In order to find modularity, the high degree of clustering and the scale-free topology under a single roof, we need to assume that modules combine with each other in a hierarchical manner.

A formal basis of the theory of hierarchical structures is given by the fact that hierarchically constrained objects are related to an ultrametric space whose geometrical image is the Cayley tree with nodes and branches corresponding to elementary cells and their links [28].
One of the first theoretical pictures [29] has been devoted to the diffusion process on either uniformly or randomly multifurcating trees. The consequent study of hierarchical structures [30] has shown that their evolution is reduced to an anomalous diffusion process in the ultrametric space that arrives at a steady-state distribution over hierarchical levels, which represents the Tsallis power law inherent to non-extensive systems [31]. Principal peculiarity of the Tsallis statistics is known to be governed by the deformed algebra [32].

Our work is devoted to the studying of creation conditions of a vast variety of hierarchical trees on the basis of the methods developed initially within quantum calculus [33]. Along this line, we restrict ourselves by considering ideal hierarchical trees which possess the underlying properties: order, predictability, and pyramidal structure [34]. These trees relate to the casual graphs, that are connected, directed acyclic graphs in which the arrows depict couplings between nodes of the nearestneighbour hierarchical levels.

The outline of the paper is as follows. In Section II, we discuss the statistical peculiarities of the picture of a hierarchical structure creation to demonstrate that effective energies of hierarchical levels remain to be additive values, while a set of corresponding probabilities becomes both non-additive and non-multiplicative due to the coupling between different levels. Section III proceeds with this discussion to formulate the connection between the probabilities to find hierarchical levels with a given set of effective energies and the probability to associate these levels into a united structure. We argue that a consistent probabilistic picture requires the use of the deformed algebra, whose main rules are stated in Appendix A. Further consideration is based on a study of the main types of hierarchical trees depicted in Fig. 1: 

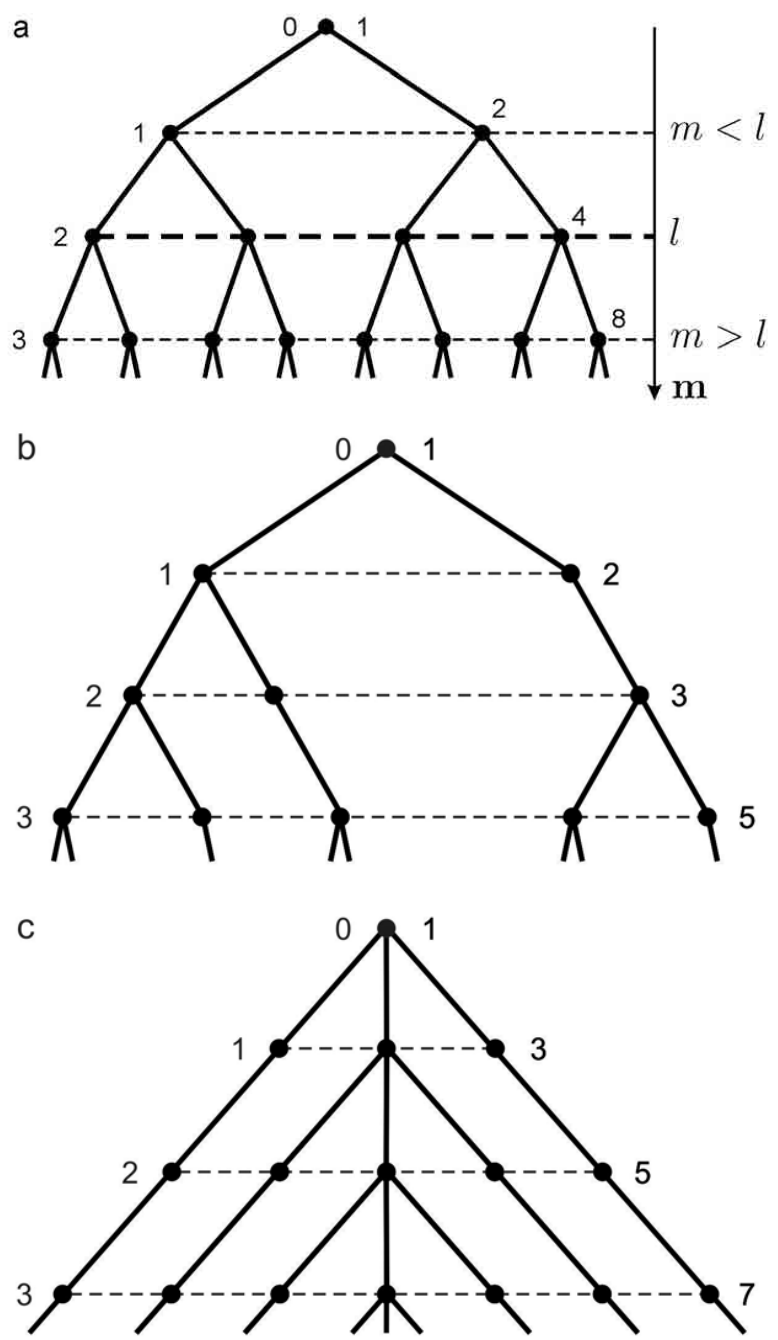

Fig. 1. The main types of hierarchical trees (level numbers and node sum are indicated left and right respectively). Top-down: regular tree with branching index $b=2$, Fibonacci tree with $b=2$, and degenerate tree with $b=3$.

Sections IV and V are devoted to the analytical definition of the creation probabilities of both regular and degenerate trees, while in Section VI we find these for both Fibonacci and scale-free trees numerically. The case of an arbitrary tree is considered in Section VII. Section VIII is devoted to the discussion of the obtained results. Appendix B contains details of calculations of the sum of terms of deformed geometrical progression that appears while considering the degenerate tree, and in Appendix $\mathrm{C}$ asymptotic forms of the distributions over hierarchical levels of both regular and degenerate trees are obtained.

\section{STATISTICAL PECULIARITIES OF HIERARCHICAL ENSEMBLES}

Standard statistical physics is known to be based on the Gibbs distribution

$$
p_{l} \propto \exp \left(-\varepsilon_{l} / \Delta\right)
$$

which determines the probabilities $p_{l}$ to have the energies $\varepsilon_{l}$, whose random values are scattered with the variance
$\Delta$. The distribution form (1) follows from the maximum principle of the Boltzmann entropy

$$
S=-\sum_{l} p_{l} \ln p_{l}
$$

According to distribution (1), the energy growth of a statistical state causes the exponentially fast decay of the corresponding probability. This distribution defines the behaviour of simple systems related to an ensemble of graphs, whose edges correspond to particles, while given graph relates to a state of the system. The volume conservation law mimics the condition of a constant number of nodes.

Although the energy is a key concept of the network optimization theory, it is not always possible to match its value to a given graph. However, basing on the heuristic ideas, it is always possible to attach an effective value of energy to some phenomenological parameter. Following the statistical theory [35], we cite below some distributions corresponding to different statistical ensembles.

Microcanonical ensemble of graphs is defined by attaching equal specific weights to every state of a system with the fixed energy $\varepsilon$ and the particle number $M$ (the other states have zero weight). Thus, the microcanonical ensemble of graphs is defined the by the weight

$$
p_{a}=n^{-1}
$$

of each of $n$ graphs $a$, which have $M$ edges with the energies $\varepsilon$.

Canonical ensemble consists of graphs with the fixed number $M$ of edges and weight

$$
p_{a}=Z^{-1} \mathrm{e}^{-\varepsilon_{a} / \Delta},
$$

where $\Delta$ and $\varepsilon_{a}$ are variance and energy of graph $a$, respectively. According to the normalization condition, the partition function reads

$$
Z=\sum_{b} \mathrm{e}^{-\varepsilon_{b} / \Delta} .
$$

For this category of graphs, the weight function is governed by the condition of minimum deviations from certain expected properties (for example, that the number of triangles composing a graph is constant).

Grand canonical ensemble of graphs is characterised by fixed values of both variance $\Delta$ and chemical potential $\mu$. With variation of the energy $\varepsilon_{a}$ and the number of edges (particles) $M_{a}$ the probability to realize a graph $a$ takes the value

$$
p_{a}=Z^{-1} \mathrm{e}^{-\left(\varepsilon_{a}-\mu M_{a}\right) / \Delta}
$$

where the partition function is written as

$$
Z=\sum_{b} \mathrm{e}^{-\left(\varepsilon_{b}-\mu M_{b}\right) / \Delta} .
$$

Along the line of the above statistical theory, one considers an ensemble of random graphs, whose probabilities are associated with the effective energies of their 
creation. Within this approach, every edge of the graph represents a particle $a$ of a many-body statistical system with the energy spectrum $\varepsilon_{a}$. As a result, the additivity of the $\varepsilon_{a}$ effective energies forces related probabilities $p_{a}$ to be multiplicative. For example, if we take an ensemble of $N$ non-interactive particles with the energies $\varepsilon_{a}, a=1,2, \ldots, N$, the probability to have the total energy $\epsilon_{N}=\sum_{a=1}^{N} \varepsilon_{a}$ is determined by the product $P_{N}=\prod_{a=1}^{N} p_{a}$. From a mathematical point of view, the above property follows directly from the exponential form of the Gibbs distribution (1).

As pointed out in the Introduction, hierarchically constrained statistical systems are not related to an exponential distribution. As a result, these systems cannot display both properties of the additivity of effective energies and multiplicativity of related probabilities simultaneously. The cornerstone of our approach is that the creation of a hierarchical structure does not break the law of energy conservation, so that the energies $\varepsilon_{l}$ of hierarchical levels remain to be additive values. On the other hand, due to the coupling between different levels the hierarchy essentially deforms the corresponding probabilities $p_{l}$, which become non-multiplicative. Taking into account the findings in [30] that the distribution over hierarchical levels is described by the Tsallis thermostatistics governed by the deformed algebra [32], it is natural to propose that the probability product should be deformed in accordance with this algebra. Most important, the deformed algebra [32] increases the creation probability of a hierarchical structure due to the coupling between different levels of the hierarchy. In addition, contrary to the approach in [35] it is convenient for our aims to consider the nodes of the hierarchical tree as particles of a statistical ensemble, while its edges represent a couplings between these particles.

The above law of the deformed multiplicativity determines the probabilities $p_{l}$ to create a set of hierarchical levels simultaneously. Another problem appears when we consider the connection between the creation probability of a given hierarchical level $l$ and the same for every node on this level. Let us consider for simplicity a regular tree, whose nodes multifurcate to generate a set of the $N_{l}$ nodes determined with the inherent probabilities $\pi=p_{0} / N_{l}$ where $p_{0}$ is their top magnitude being a normalization constant. If one permits additivity of the node probabilities, we arrive at the total probability of the $l$ th level realization to be independent of their numbers: $p_{l}:=N_{l} \pi=p_{0}$. Since the probability $p_{l}$ to create a hierarchical level should decay with the level number $l$, we are forced again to replace the trivial additive connection of the level probability $p_{l}$ with the node value $\pi$ by a deformed sum. As will be shown in Section IV, the deformed sum proposed in Ref. [32] takes the values which are less than usual, so that the level creation probability decays with the $l$ number of the hierarchical level.

Finally, since the creation probabilities of the hierarchical levels go beyond the probabilities related to nonhierarchical structures, the standard normalization condition based on the use of the usual sum will be broken as well. As a result, one can anticipate that the appearance of hierarchical coupling should deform the normalization condition. Due to the property of the deformed sum such a deformation should restore this condition for an arbitrary set of the creation probabilities of the hierarchical levels.

\section{FORMULATION OF THE PROBLEM}

As was mentioned above, self-similar distributions are described by the power law $p(k) \propto k^{-\gamma}$ with an exponent $\gamma>0$. This form is caused by the fact that a random variation of the node order $k$ is scale-free. In fact, the substitution of the variable $k$ by the value $k / a$ scaled by a positive constant $a$ keeps the power-law form. Historically, self-similar ensembles were used firstly in the studying of critical phenomena [36], when groups of $b$ spins ( $b=2$ corresponds to the simplest case of a binary tree) associate in clusters, then groups of $b$ clusters sequentially create a supercluster of the next level and so on, until the maximum cluster is created, which contains virtually all spines. As a result, such structure have a hierarchical character, which can be depicted by the Cayley tree (Fig. 1).

Apparently, the behaviour of a whole hierarchical system is defined by the cluster structure of all its levels, however, the property of self-similarity allows us to confine ourselves to setting a minimal cluster structure and fixing a level number. Since the hierarchical tree is a geometrical image of the ultrametrical space [28], a description of the complex network within the generation method reduces to the description of a diffusion process in this space [30].

Following the line of Ref. [15], let us show that the evolution of a self-similar complex system represents an anomalous diffusion over hierarchical levels, which leads to a stationary distribution in the Tsallis form. For this purpose, we consider a probability destiny $p_{\mathrm{u}}=p_{\mathrm{u}}(t)$ of the system distribution over the ultrametric space coordinates $\mathrm{u}$ at the moment of time $t$. This distribution is governed by the kinetic master equation [30]

$$
\tau \dot{p}_{\mathrm{u}}=\sum_{\mathrm{u}^{\prime}}\left(f_{\mathrm{uu}^{\prime}} p_{\mathrm{u}^{\prime}}-f_{\mathrm{u}^{\prime} \mathrm{u}} p_{\mathrm{u}}\right)
$$

where the dot stands for the time differentiation, $\tau$ is a relaxation time, $f_{\mathrm{uu}^{\prime}}$ represents the transition rate from $\mathrm{u}^{\prime}$ to $\mathrm{u}$. In order to find the form of dependencies on ultrametric coordinates let us consider a regular hierarchical tree, which is characterized by the fixed branching index $b>1$ and the variable number of hierarchical levels $l \gg 1$. In so doing, the ultrametric coordinate $u$ and is $l$-digit figure in the $b$-nary number system: $\mathrm{u} \equiv u_{0} u_{1} \ldots u_{m} \ldots u_{l-1}, u_{m}=0,1, \ldots, b-1$. Accordingly, the transition rate is written as a power series $f_{\mathrm{uu}^{\prime}}=\sum_{m=0}^{n} f\left(u_{m}-u_{m}^{\prime}\right) b^{l-m}$ where the first term $(m=0)$ relates to the top level of hierarchy which defines the whole system behaviour, whereas the last one $(m=l)$ relates to the lowest level which corresponds to the smallest clusters. According to the definition, the distance between points $\mathrm{u}$ and $\mathrm{u}^{\prime}$ is $0 \leq s \leq n$ if conditions 
$u_{m}=u_{m}^{\prime}$ for $m=0,1, \ldots, l-(s+1)$ and $u_{m} \neq u_{m}^{\prime}$ for $m=l-s, l-s+1, \ldots, l$ are fulfilled [28]. Thus, when the distance $s$ is fixed, the first $l-s$ terms of the above series equal zero by definition, while the last ones, whose number equals $s$, contain the factor $b^{l-m}$ of the value, being in the continuous limit $b \rightarrow \infty$ much less than the factor $b^{s}$ that is the first of the other terms. As a result, only the term $m=l-s$ with $f_{\mathrm{uu}^{\prime}} \sim b^{s}=b^{l-m}$ is significant in the considered power series. It can be similarly shown that the probability destiny is $p_{\mathrm{u}} \sim b^{l-s}=b^{m}$. In the case of a random tree the branching index $b$ becomes variable, which leads to the transition rate $f_{\mathrm{uu}^{\prime}} \Rightarrow f_{l-m}$ and the probability destiny $p_{\mathrm{u}} \Rightarrow p_{m}$ takes the form of the Mellin transform [37]

$$
f_{l-m}=\int_{0}^{\infty} f(b) b^{l-m} \mathrm{~d} b, \quad p_{m}=\int_{0}^{\infty} p(b) b^{m} \mathrm{~d} b,
$$

where $f(b)$ and $p(b)$ are the corresponding weight functions.

As a result, the kinetic master equation is written as follows:

$$
\tau \dot{p}_{l}=\sum_{m>l} f_{m-l} p_{l}-\sum_{m<l} f_{l-m} p_{m} .
$$

In contrast to the form (8) related to the continuous ultrametric space, here we pass to the corresponding discrete representation, being related to hierarchical trees whose simplest types are depicted in Figure 1. The first term of the right-hand side of Eq. (10) takes into account the hierarchical couplings of the fixed level $l$ with the nodes of lower levels $m>l$, while the second term makes the same for upper levels $m<l$. In comparison with usual systems, both the above terms have the opposite signs because it is inherent in the autonomous hierarchical systems to restore spontaneously instead of going to decay.

Expanding the probability $p_{m}$ in a series over the difference $l-m$, one obtains in the limit $l \gg 1$

$$
\tau \dot{p}_{l}=-D \frac{\partial^{2} p_{l}}{\partial l^{2}}+\mathcal{D}_{l} p_{l}
$$

where the lower moments $\sum_{m<l}(l-m) f_{l-m}=0$ and $2 D=\sum_{m<l}(l-m)^{2} f_{l-m}$ are taken into account; the operator

$$
\mathcal{D}_{l}:=\sum_{m>l} f_{m-l}-\sum_{m<l} f_{l-m}
$$

determines a difference between the transition rates from the $l$ th level onto the lower and upper levels. Usually, the conditions $m>l$ and $m<l$, taking place in the sums of Eqs. (10) and (12), are absent, and $\mathcal{D}_{l}=0$. However, one has no reason to take the condition $\mathcal{D}_{l}=0$ for the hierarchical systems because the transition rates depend essentially on the levels $m$ (to be lower or upper ones) which are coupled with the given hierarchical level $l$.

As is mentioned in the Introduction, we consider only ideal hierarchical trees, whose coupled nodes belong to the nearest-neighbour hierarchical levels. In this case, the transition rates in Eqs. (10) and (12) take non-zero values $f_{m-l}=f_{l}$ at $m=l+1$ and $f_{l-m}=f_{l}$ at $m=l-1$. Then, within the steady state where $\dot{p}_{l}=0$, the master equation (10) arrives at the relation

$$
\frac{f_{l-1}}{f_{l}}=\frac{p_{l}}{p_{l-1}}
$$

that allows one to express the transition rates through the stationary probabilities. Basing on the independent combinatorial approach, we will obtain in Section 4 distribution (26) over hierarchical levels of the regular tree, being the simplest example of hierarchical graphs. With using this distribution we find

$$
\begin{aligned}
\frac{f_{l-1}}{f_{l}} & =\frac{\left[1+(1-q) b^{-l}\right]_{+}^{b^{l}}-1}{\left[1+(1-q) b^{-(l-1)}\right]_{+}^{b^{l-1}}-1} \\
& \simeq 1-\frac{1}{2} \frac{(q-1)^{2}}{\mathrm{e}^{q-1}-1} b^{-(l-1)}
\end{aligned}
$$

where the last estimation corresponds to the limit $b \rightarrow \infty$ related to a continuons ultrametric space. So, one can conclude that the transition rates decay exponentially fast from top to bottom of a regular tree. As the asymptotic form (44) shows, such behaviour is kept with the passage to the degenerate tree.

As a fact of fundamental importance it is worth noting that the probability distribution (26) can be written in the form of (27) expressed trough the basic number (28). Since the latter represents the eigenvalue of the Jackson derivative acting on the homogeneous function as the eigenfunction [33], one can suppose that operator (12) which, in accordance with relation (13), is expressed through a combination of related probabilities should be determined by the Jackson derivative being an archetype of self-similar hierarchical systems [38]. As a result, we propose to use the ansatz

$$
\mathcal{D}_{l}:=-d q p_{l}^{q-1} \frac{\partial}{\partial l}
$$

with the positive parameters $q, d$; the formal basis for such an ansatz is that the integral $\int_{l}^{q l} \mathcal{D}_{l} p_{l} \mathrm{~d} l$ is reduced, accurate within the factor $-d(q-1)$, to the Jackson derivative $D_{l} p_{l}^{q}:=\frac{p_{q l}^{q}-p_{l}^{q}}{q-1}$. Then, the master equation (11) takes the final form

$$
\tau \dot{p}_{l}=-\frac{\partial}{\partial l}\left(d p_{l}^{q}+\frac{\partial}{\partial l} D p_{l}\right)
$$

The steady-state solution of this equation has the form

$$
\begin{aligned}
p_{l} & =\left(p_{0}^{-(q-1)}+\frac{q-1}{\Delta} l\right)^{-\frac{1}{q-1}} ; \quad p_{0} \equiv\left(\frac{2-q}{\Delta}\right)^{\frac{1}{2-q}}, \\
\Delta & \equiv D / d
\end{aligned}
$$

With growing the level number $l$, the creation probability $p_{l}$ of a hierarchical cluster decreases monotonically from the maximum value $p_{0}$ on the upper level $l=0$ related to the whole system. 
With the use of the deformed exponential (A.1) and the effective energy $\varepsilon_{l}=\left(\frac{2-q}{\Delta}\right)^{\frac{q-1}{2-q}} l$, the creation probability (17) of the $l$ th hierarchical level takes the canonical form

$$
p_{l}=p_{0} \exp _{q}\left(-\frac{\varepsilon_{l}}{\Delta}\right)
$$

related to the Tsallis statistics [31]. According to the consideration in [39], the effective temperature $\Delta$ obeys standard thermodynamic relations under the condition that a random variable $\phi_{l}$ distributed over the levels of a hierarchical self-similar set is defined by the $q$-normalized average $\langle\phi\rangle=\sum_{l} \phi_{l} \mathcal{P}_{l}$, which is based on the use of the escort probability $\mathcal{P}_{l}:=\frac{p_{l}^{q}}{\sum_{l} p_{l}^{q}}$ instead of the initial distribution $p_{l}$. The determination of such a type of averages arrives at a separate problem which will be considered elsewhere [40].

As was pointed out in the previous section, with the growth of the difference $|q-1|$ probability (18) increases at arbitrary values of the energy $\varepsilon_{l}$ with respect to the non-deformed value related to the parameter $q=1$. On the other hand, the deformed sum (A.3) decreases with the growing the parameter $q>1$. As a result, one can anticipate that a self-consistent probabilistic picture of hierarchical ensembles is reached, if one proposes that the normalization condition

$$
p_{0} \oplus_{q} p_{1} \oplus_{q} \cdots \oplus_{q} p_{n}=1, \quad q>1
$$

is deformed to fix the top level probability $p_{0}$ according to the summation rule (A.3). We will show in Sections IV and $\mathrm{V}$ that the analytical consideration of both regular and degenerate hierarchical trees on the basis of the above propositions appears to be self-consistent.

In accordance with the proposed statistical picture, we suppose that the total energy $\epsilon_{n}$ of a hierarchically constrained statistical ensemble comprising of the $n>1$ levels $l=0,1, \ldots, n$ is connected with the effective energies $\varepsilon_{l}$ of the level creation by the usual sum

$$
\epsilon_{n}:=\sum_{l=0}^{n} \varepsilon_{l} .
$$

Within the statistical theory of random networks [35], effective energies $\varepsilon_{l}$ are reduced to a constant for the microcanonical ensemble and are fixed by the set of given probabilities $p_{l}$ according to the relation $\varepsilon_{l}=$ $-\Delta \ln \left(p_{l}\right)+$ const for both canonical and grand canonical ensembles with the effective temperature $\Delta$. The above considered master equation (16) leads to the Tsallis distribution (18) with the linearly dependent effective energy $\varepsilon_{l}=\left(\frac{2-q}{\Delta}\right)^{\frac{q-1}{2-q}} l$.

In opposition to the statistical theory [35] based on the Gibbs distribution, we are based on the Tsallis one, so that probability $P_{n}$ to create an $n$-level hierarchical structure should be connected with the total energy $\epsilon_{n}$ by means of the relation $\epsilon_{n}=-\Delta \ln _{q}\left(P_{n}\right)$ using the deformed logarithm (A.1). Then, condition (20) leads to the additivity of these logarithms:

$$
\ln _{q}\left(P_{n}\right)=\sum_{l=0}^{n} \ln _{q}\left(p_{l}\right) .
$$

In accordance with the first rule (A.5), this equation gives the probability relation

$$
P_{n}:=p_{0} \otimes_{q} p_{1} \otimes_{q} p_{2} \otimes_{q} \cdots \otimes_{q} p_{n} .
$$

Thus, in contrast to ordinary statistical systems, the creation probability $P_{n}$ of a hierarchical structure equals the deformed production of specific probabilities $p_{l}$ related to levels $l=0,1, \ldots, n$. As the product definition (A.2) shows, growth of the deformation parameter $q>1$ increases essentially the probability (22) in comparison with the usual value at $q=1$. From a physical point of view, the above deformation of the factorization rule for independent probabilities recovers the additivity condition (20) for the corresponding energies within the Tsallis' thermostatistics.

Taking into account (A.1), Eq. (21) gives the explicit form of the creation probability of a hierarchical structure:

$$
\begin{aligned}
P_{n} & =\exp _{q}\left[\frac{\sum_{l=0}^{n} p_{l}^{1-q}-(n+1)}{1-q}\right] \\
& =\left(\sum_{l=0}^{n} p_{l}^{1-q}-n\right)_{+}^{\frac{1}{1-q}} .
\end{aligned}
$$

Here, the last expression follows directly from the deformed production (22) taking into account rule (A.2). Relations (23) imply a decrease in the creation probability with the growth of the hierarchical tree in accordance with the difference equation

$$
P_{n-1}^{1-q}-P_{n}^{1-q}=1-p_{n}^{1-q} .
$$

In the non-deformed limit $q \rightarrow 1$, relations (22) and (23) are reduced to the ordinary rule $P_{n}=\prod_{l=0}^{n} p_{l}$ (respectively, Eq. (24) reads $P_{n} / P_{n-1}=p_{n}$ ), while at $q=2$ the creation probability $(23)$ takes a maximal value.

It is worthy to note that the principle peculiarity of the above scheme is that the level energies $\varepsilon_{l}$ remain to be additive values because the creation of a hierarchical structure does not break the law of energy conservation. However, the hierarchy deforms essentially the probability relations (19), (22), (23) and (24) due to the appearance of the coupling between level probabilities $p_{l}$.

According to Eq. (23) the consequent step in the definition of the creation probability $P_{n}$ of a hierarchical structure is the determination of a set of probabilities $\left\{p_{l}\right\}_{0}^{n}$ related to different hierarchical levels. Let us consider first the simplest case of the regular tree depicted in Fig. 1(a).

\section{REGULAR TREE}

Let us consider a regular tree whose nodes multifurcate on a certain level $l$ with the constant branching index 
$b>1$ to generate a set of the $N_{l}=b^{l}$ nodes determined with inherent probabilities $\pi=p_{0} / N_{l}=p_{0} b^{-l}$ where $p_{0}$ is their top magnitude being a normalization constant. Within a naive proposition, one could permit additivity of the node probabilities to arrive at the total probability of the $l$ th level realization to be $p_{l}:=N_{l} \pi=p_{0}$. Thus, within the condition of additivity of the node probabilities, related values $p_{l}=p_{0}=(n+1)^{-1}$ for all levels appear to be non-dependent of their numbers $l=0,1, \ldots, n$.

To escape such a trivial situation we propose to replace the above additive connection of the level probability $p_{l}$ with the node value $\pi$ by the following deformed equalities:

$$
\frac{p_{l}}{p_{0}}:=\underbrace{\frac{\pi}{p_{0}} \oplus_{q} \frac{\pi}{p_{0}} \oplus_{q} \cdots \oplus_{q} \frac{\pi}{p_{0}}}_{N_{l}} \equiv N_{l} \odot_{q} \frac{\pi}{p_{0}}=b^{l} \odot_{q} b^{-l} .
$$

Presenting here the deformed sum of $N_{l}$ identical terms with the help of formula (A.4), one obtains required level distribution in the binomial form

$$
p_{l}=\frac{\left[1+(1-q) b^{-l}\right]_{+}^{b^{l}}-1}{1-q} p_{0} .
$$

A much more elegant form

$$
p_{l}=b^{-l}\left[b^{l}\right]_{\lambda_{l}} p_{0}, \quad \lambda_{l} \equiv\left[1+(1-q) b^{-l}\right]_{+}
$$

can be attached to equality (26) using the basic number [33]

$$
[n]_{\lambda}:=\frac{\lambda^{n}-1}{\lambda-1} .
$$

According to Appendix $\mathrm{C}$, in the limit $b \rightarrow \infty$ related to the continuous ultrametric space [28] distribution (26) is simplified into the form

$$
p_{l} \simeq p_{\infty}+\frac{p_{0}}{2} \frac{q-1}{\mathrm{e}^{q-1}} b^{-l}
$$

that shows exponentially fast decay with the growth of the level number $l \geq 1$. Here, the limit value

$$
p_{\infty}=\frac{\mathrm{e}^{1-q}-1}{1-q} p_{0}=p_{0} \ln _{q} \mathrm{e}
$$

is introduced to be $p_{\infty}>p_{0}$ at the deformation $q<1$ and $p_{\infty}<p_{0}$ at $q>1$. Inserting Eq. (30) into Eq. (23) leads to the following expression for the creation probability of a regular tree:

$$
P_{n}=\left\{p_{0}^{1-q}\left[1+n\left(\ln _{q} \mathrm{e}\right)^{1-q}\right]-n\right\}^{\frac{1}{1-q}}
$$

Respectively, the deformed normalization condition related to the limit $b \gg 1$ takes the form

$$
\begin{aligned}
& p_{0} \oplus_{q}\left[n \odot_{q}\left(p_{0} \ln _{q} \mathrm{e}\right)\right] \\
= & \frac{\left[1+p_{0}(1-q)\right]\left[1+p_{0}(1-q) \ln _{q} \mathrm{e}\right]^{n}-1}{1-q}=1 .
\end{aligned}
$$

In accordance with the above consideration, Fig. 2(a) shows probability (26) increasing with the growing number $l$ of a hierarchical level at $q<1$ and decays at $q>1$.
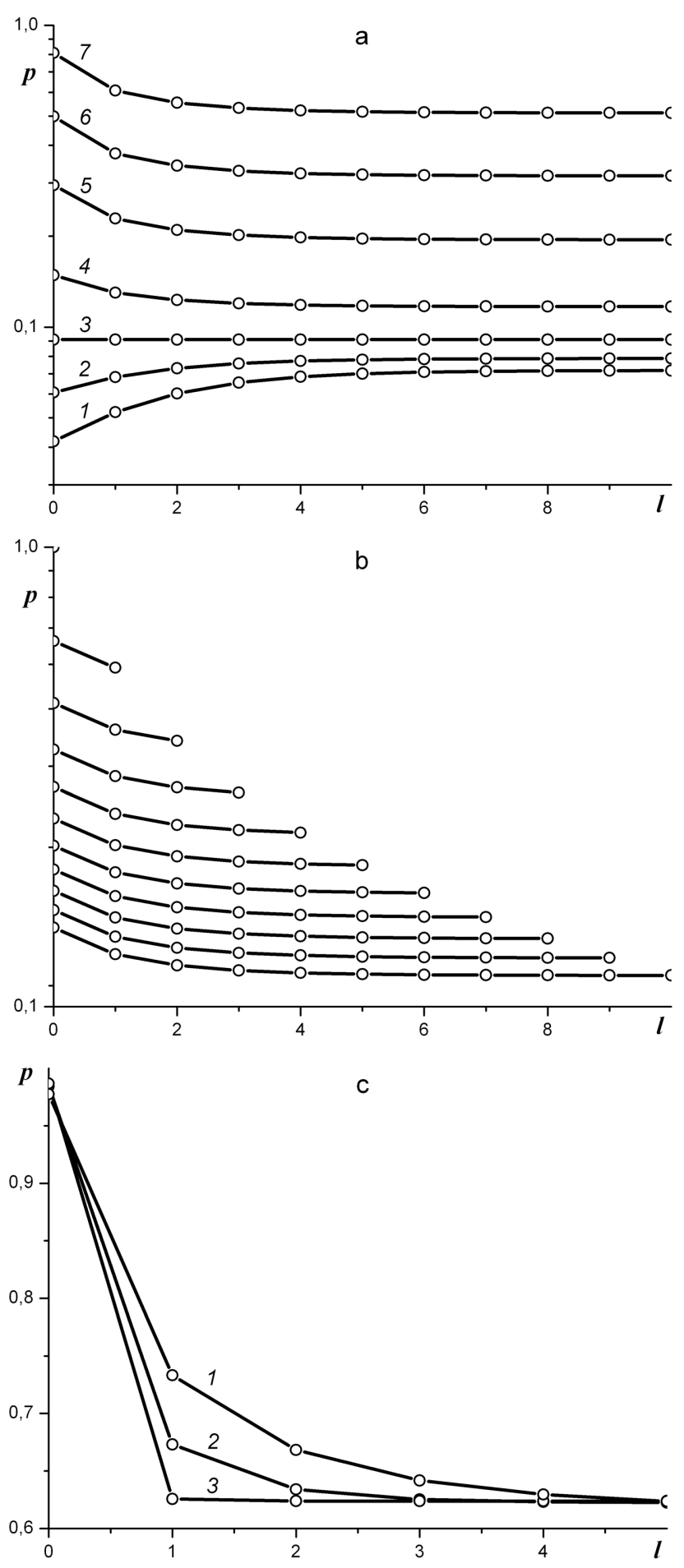

Fig. 2. Probability distribution over hierarchical levels of the regular tree as function of the level number at: (a) $b=2$, $n=10$ and $q=10^{-4}, 0.5,1.0,1.5,1.9,1.99,1.9999$ (curves $1-7$, respectively); (b) $b=2, q=1.5$ and $n=1,2, \ldots, 10$ (curves top-down, respectively); (c) $q=1.9999, n=5$ and $b=2,4,100$ (curves $1-3$, respectively).

From a physical point of view, the creation probability of a lower hierarchical level should be less than for the higher levels, so that one ought to conclude that only the case $q>1$ is meaningful. 
In this case, with the growing total number of hierarchical levels $n$, the probability distribution (26) normalized with condition (19) decays as shown in Fig. 2(b). Characteristically, the form of this distribution depends very weakly on both deformation parameter $q$ and branching index $b$ excluding the domain $2-q \ll 1$. According to Fig. 2(c), within this domain, the probability distribution over hierarchical levels decays not so fast at small values of the branching index $b$. With a large growth of the parameter $b \gg 1$, the dependence $p_{l}$ decreases faster to reach exponentially fast the minimum value (30) that is independent of the branching index $b$.
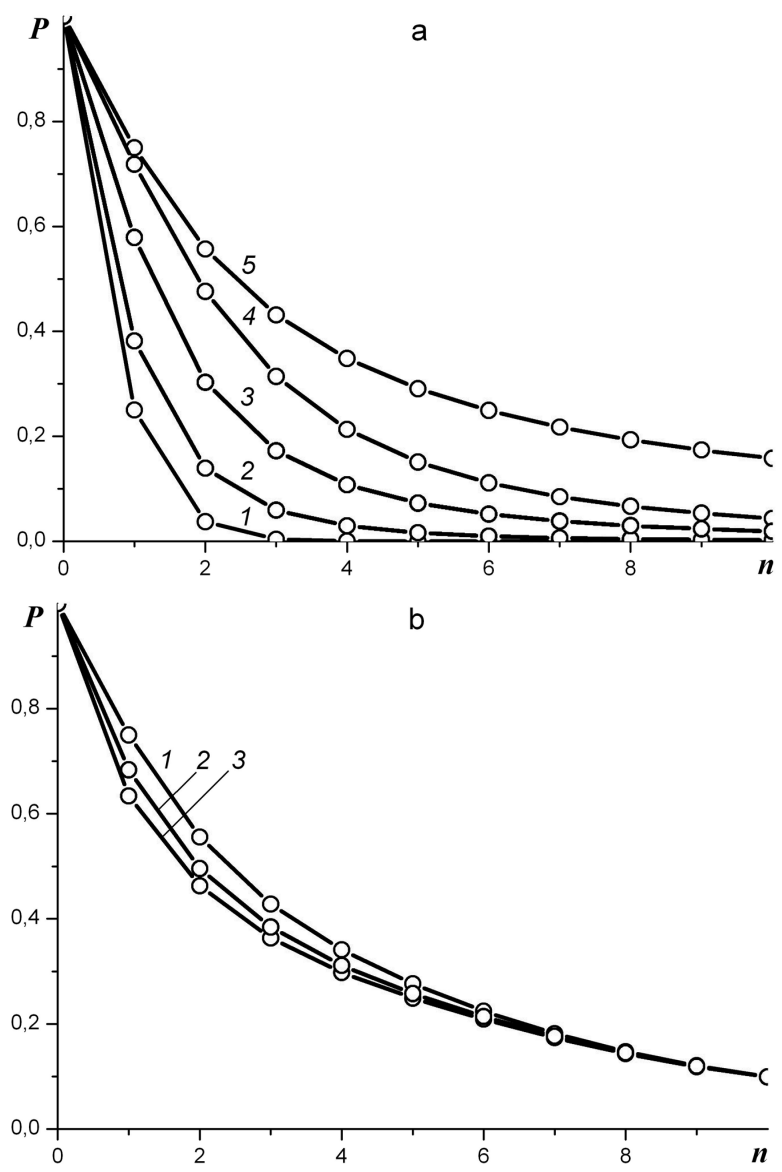

Fig. 3. Creation probability of the regular hierarchical tree in dependence of the whole number of its levels at: (a) $b=2$ and $q=1.0001,1.5,1.9,1.99,1.9999$ (curves $1-5$, respectively); (b) $q=1.9999, n=10$ and $b=2,4,100$ (curves $1-3$, respectively).

As numerical calculations show, the creation probability (23) takes meaningful values $P_{0} \leq 1$ for the deformation parameters $q>1$ only. According to Fig. 3(a) the dependence of this probability on the whole number of tree levels slows down monotonically with a decaying rate that decreases considerably only near the limit value $q=2$. On the other hand, Fig. 3(b) shows that a variation of the branching index $b \gg 1$ affects appreciably the dependence of the the creation probability only for moderate numbers of tree levels within the domain $2-q \ll 1$.
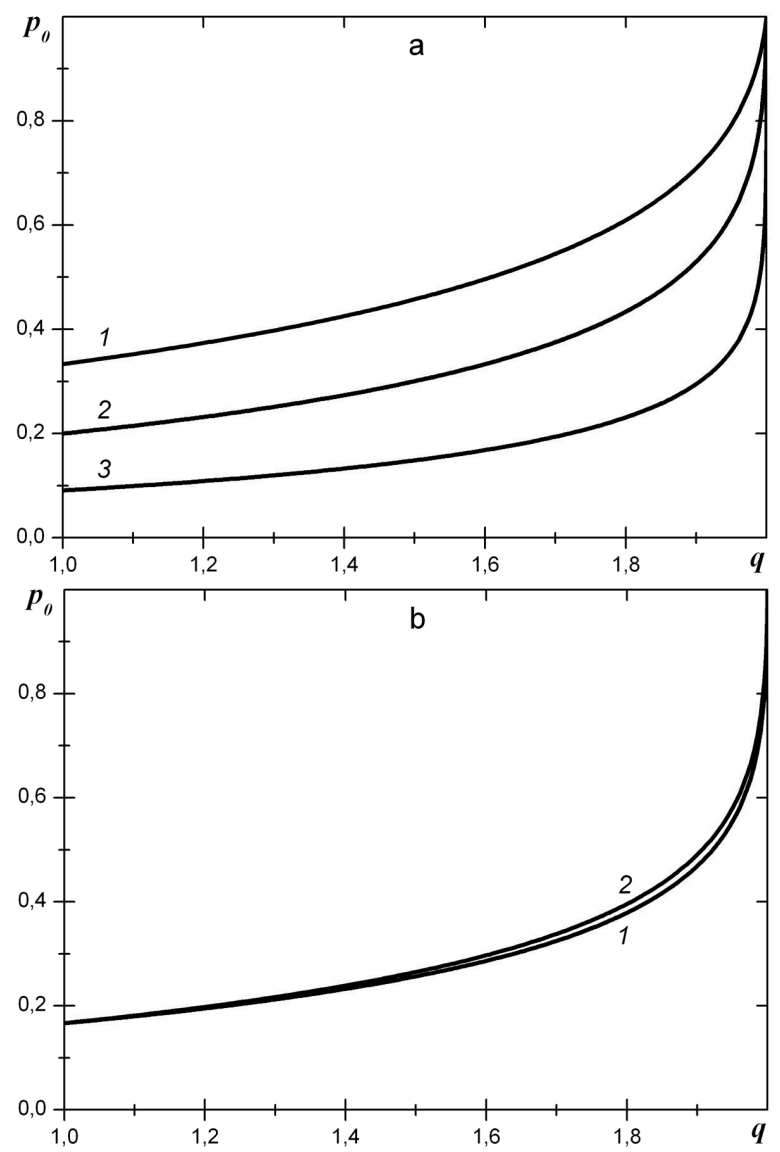

Fig. 4. Top level probability of the regular tree as function of the deformation parameter at: (a) $b=2$ and $n=2,4,10$ (curves $1-3$, respectively); (b) $n=5$ and $b=2,10^{3}$ (curves 1,2 , respectively)

The above data indicate a distinctive feature in the behaviour of the regular hierarchical tree near the limit value $q=2$ where the dependence (26) has no singularity. This feature is corroborated with the dependence of the top level probability on the deformation parameter depicted in Fig. 4. It is aparent, independent of both the total number of levels $n$ and

branching index $b$, of the type shown in Fig. 2(a) this probability increases monotonically with the $q$-growth to reach sharply the limit value $p_{0}=1$ in the point $q=2$. Obviously, this means anomalous increasing probabilities $p_{l}$ for the whole set of hierarchical levels (that with the curve 7 ). Though, within the domain $2-q \ll 1$, the ordinary normalization condition $\sum_{l=0}^{n} p_{l}=1$ is violated appreciably, definition (A.3) shows that the deformed normalization condition (19) can be recovered at a large parameter $q$. However, beyond the border $q=2$ this condition is not satisfied at all. As a result, we arrive at the conclusion that physically meaningful values of the deformation parameter are concentrated within the domain $q \in[1,2]$.

\section{DEGENERATE TREE}

As shown in Fig. 1, the difference between regular and degenerate trees is that all nodes multifurcate on each 
level in the former case, while the only one node branches in the latter. In this sense, the degenerate tree can be considered as an antipode of the regular one to be studied analytically.

According to Fig. 1(b), on the $l=1$ level, the branching process with the index $b>1$ creates $N_{1}=b$ nodes with equal probabilities $b^{-1}$. Next, on the $l=2$ level, $b-1$ nodes out of $N_{2}=2(b-1)+1$ ones have the same probabilities $b^{-1}$, while the other $b$ nodes relate to the smaller value $b^{-2}$. On the $l=3$ level, out of $N_{3}=3(b-1)+1$ nodes one has $b$ nodes with probabilities $b^{-3}, b-1$ with $b^{-2}$ and $b-1$ with $b^{-1}$. Hence, on the $l$ th level $N_{l}=l(b-1)+1$ nodes are partitioned into $l$ groups, among which $l-1$ ones contain $b-1$ nodes with the probabilities $b^{-1}, b^{-2}, \ldots, b^{-(l-1)}$, while the last group has $b$ nodes with equal probabilities $b^{-l}$. Taking into account such a partitioning, the creation probability of the $l$ th hierarchical level is expressed with the following relations:

$$
\begin{aligned}
& \frac{p_{l}}{p_{0}}=\underbrace{\left[(b-1) \odot_{q} b^{-1}\right] \oplus_{q} \cdots \oplus_{q}\left[(b-1) \odot_{q} b^{-(l-1)}\right]}_{l-1} \\
& \oplus_{q}\left(b \odot_{q} b^{-l}\right) \\
& =\underbrace{\left[(b-1) \odot_{q} b^{-1}\right] \oplus_{q} \cdots \oplus_{q}\left[(b-1) \odot_{q} b^{-l}\right]}_{l} \oplus_{q} b^{-l} \\
& :=\left[(b-1) \odot_{q}\left(S_{l+1} \ominus_{q} 1\right)\right] \oplus_{q} b^{-l} .
\end{aligned}
$$

Here, in the last equation the sum of the deformed geometrical series

$$
S_{l}:=\underbrace{1 \oplus_{q} b^{-1} \oplus_{q} b^{-2} \oplus_{q} \cdots \oplus_{q} b^{-(l-1)}}_{l}
$$

is introduced. As a related consideration in Appendix B shows, this sum is expressed by the power series

$$
S_{l}=\sum_{k=0}^{l-1} C_{l}^{k+1}(b)(1-q)^{k} b^{-\frac{k(k+1)}{2}}
$$

with the deformed binomial coefficients [33]

$$
C_{l}^{k}(b) \equiv \prod_{m=0}^{k-1} \frac{1-b^{-(l-m)}}{1-b^{-(m+1)}} .
$$

Inserting Eq. (35) into the last relation (33), one obtains the final expression for the $l$ th level creation probability

$$
p_{l}=\frac{\left[1+(1-q) b^{-l}\right]\left[1+(1-q) \Sigma_{l}\right]^{b-1}-1}{1-q} p_{0}
$$

where one denotes

$$
\Sigma_{l} \equiv S_{l+1} \ominus_{q} 1=\frac{1}{2-q} \sum_{k=1}^{l} C_{l+1}^{k+1}(b)(1-q)^{k} b^{-\frac{k(k+1)}{2}} .
$$

Within the product representation

$$
S_{l}=\frac{1}{1-q}\left\{\prod_{m=0}^{l-1}\left[1+(1-q) b^{-m}\right]-1\right\}
$$

one has

$$
\Sigma_{l}=\frac{1}{1-q}\left\{\frac{\prod_{m=0}^{l}\left[1+(1-q) b^{-m}\right]}{2-q}-1\right\} .
$$

Then probability (37) takes the explicit form

$$
p_{l}=\frac{\left[1+(1-q) b^{-l}\right] \prod_{m=1}^{l}\left[1+(1-q) b^{-m}\right]^{b-1}-1}{1-q} p_{0} .
$$

This expression can be rewritten in a more elegant form

$$
p_{l}=\frac{\left(1+\frac{1-q}{b^{l}}\right)\left(1+\frac{1-q}{b}\right)_{1 / b}^{(b-1) l}-1}{1-q} p_{0}
$$

using the basic-deformed binomial

$$
(x+y)_{\mu}^{a n}:=\prod_{m=1}^{n}\left(x+\mu^{m-1} y\right)^{a}
$$

modified by exponent $a$ for arbitrary variables $x$ and $y$. It is interesting to note that in the case of the regular tree the simplest form (27) of the probability distribution is achieved if one uses the basic number (28), while the same simplification for the degenerate tree requires using the basic-deformed binomial (43). Both mathematical constructions (28) and (43) are known to represent paradigmatic conceptions of the quantum calculus [33].

As is shown in Appendix C, in the limit $b \rightarrow \infty$ related to continuous ultrametric space, the probability distribution (42) takes the asymptotic form

$$
p_{l} \simeq p_{\infty}+\frac{p_{0}}{2} \frac{q-1}{\mathrm{e}^{q-1}} b^{-2 l}
$$

Similarly to the case of the regular tree characterized by asymptotics (29) this distribution decays exponentially fast to the limit probability $p_{\infty}$ determined by Eq. (30). In the limit $q \rightarrow 1$ related to the slight hierarchy, the factor standing in Eq. (44) before $b^{-2 l}$ is simplified to the form $q-1$. Thus, one can conclude that both regular and degenerate trees display the same behaviour. Besides, in spite of apparent differences between formulas (26) and (41), direct calculations show actually coincident forms of the probability distributions over hierarchical levels of these trees. We postpone a numerical study of the creation probability for the degenerate tree to the following section where consideration of the free-scale tree allows us to compare all the results obtained analytically.

\section{FREE-SCALE TREE}

Above, we have considered two conceptual examples of hierarchical trees with a self-similar structure - regular and degenerate trees depicted in Fig. 1. In this section, 
we study a free-scale tree whose structure is rather random, but the probability distribution over hierarchical levels tends to the power-law form inherent in self-similar statistical systems [41].
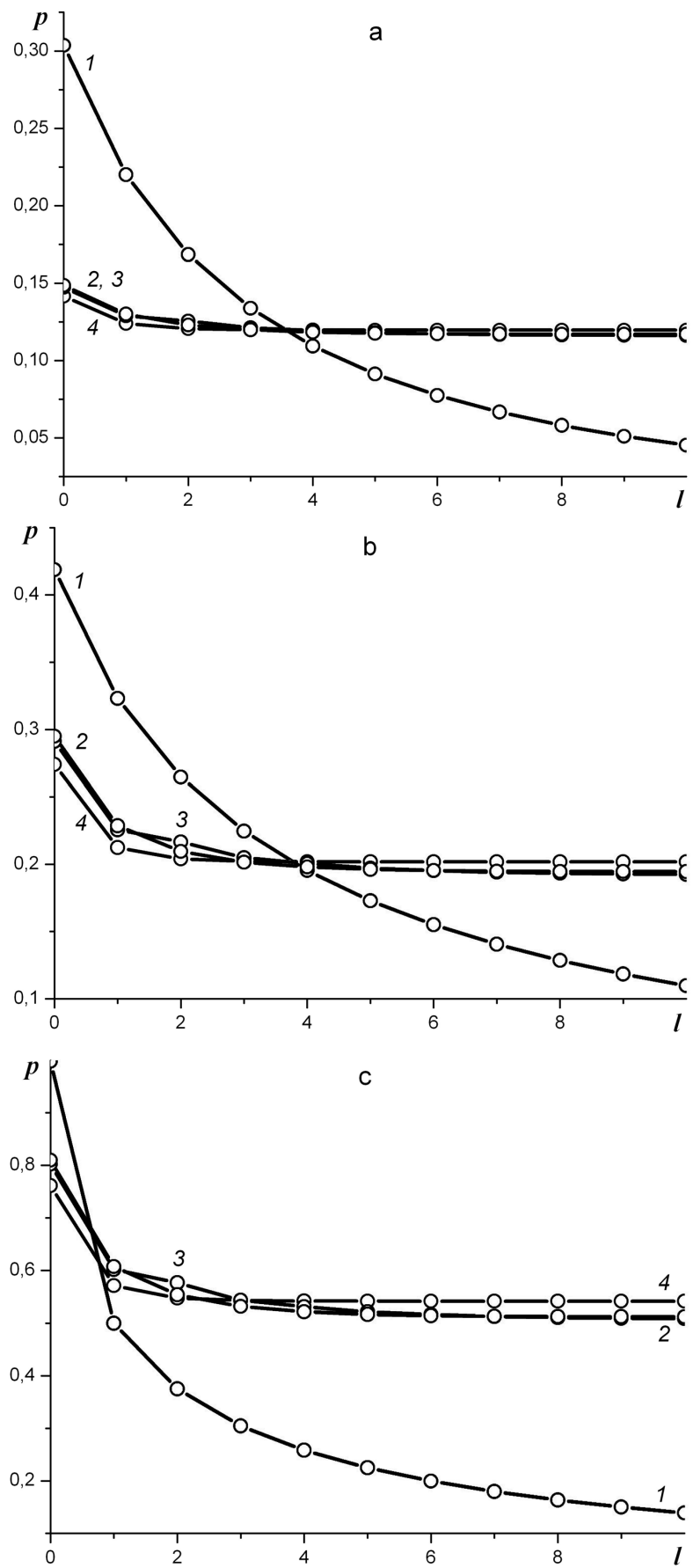

Fig. 5. Probability distributions over hierarchical levels for free-scale, regular, Fibonacci and degenerate trees (curves $1-4$, respectively) at $\Delta=2, b=2, n=10$ and $q=1.5$ (a), $q=1.9$ (b) and $q=1.9999$ (c).

In this case, the probability distribution over tree levels is determined by the discrete difference equation [30]

$$
p_{l+1}-p_{l}=-p_{l}^{q} / \Delta, \quad l=0,1, \ldots, n
$$

accompanied with the deformed normalization condition (19) ( $\Delta$ being a distribution dispersion). It is easy to show that at the continuum limit $l \rightarrow \infty$ equation (45) leads to a power-law dependence (17).
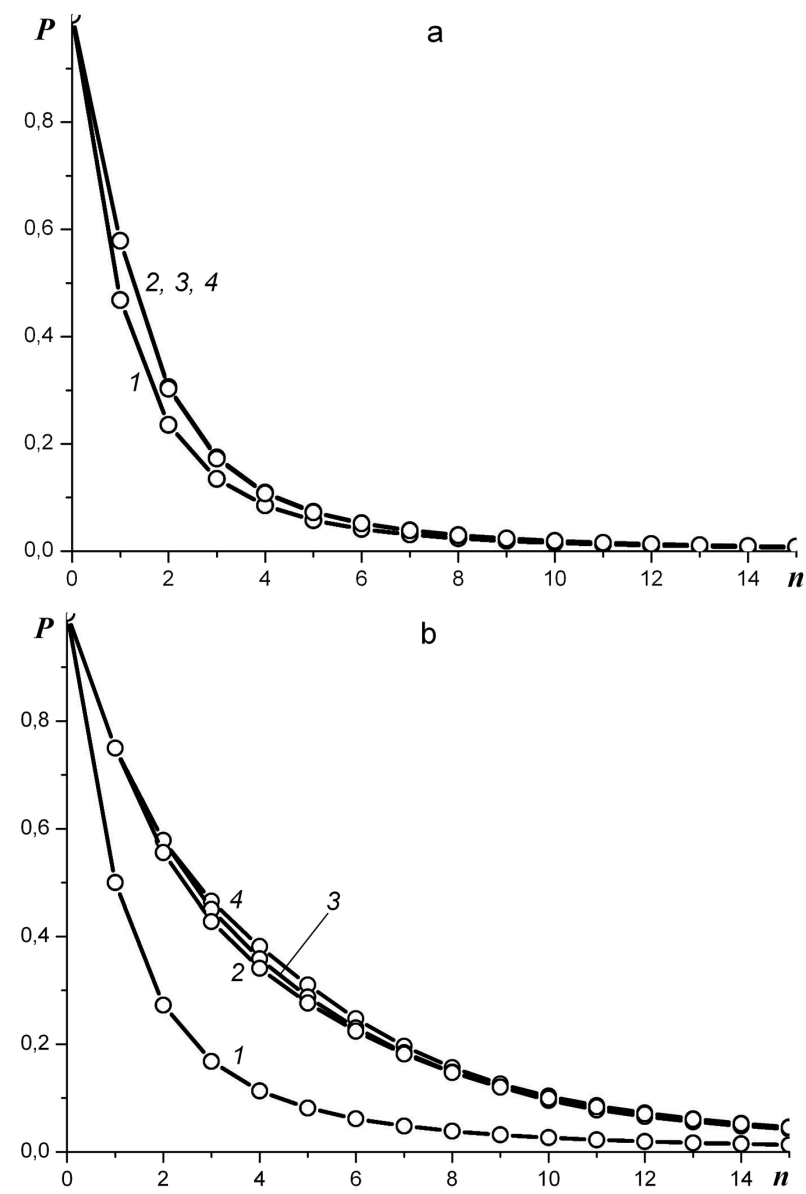

Fig. 6. Creation probabilities of free-scale, regular, Fibonacci and degenerate hierarchical trees (curves 1-4, respectively) as function of the whole level number at $\Delta=2, b=2$ and $q=1.9$ (a) and $q=1.9999$ (b).

In Figs. 5 we compare the probability distributions over hierarchical levels of free-scale, regular and degenerate trees at different values of the deformation parameter. It is seen at all $q$-values that form of these distributions is actually equal for regular and degenerate trees, but differs appreciably for free-scale tree, where the level probability falls down much more strongly, than for both other trees. In accordance with such behaviour, the creation probabilities depicted in Figs. 6 decays faster for the free-scale tree than in the case of the regular and degenerate ones. Characteristically, this difference appears only within the domain $2-q \ll 1$ of the deformation parameter variation.

As shown in the end of section III, such behaviour is stipulated by the singular dependence of the top level probability $p_{0}$ on the deformation parameter near the point $q=2$. According to Fig. 7 this singularity is inherent in of the all considered hierarchical trees. 


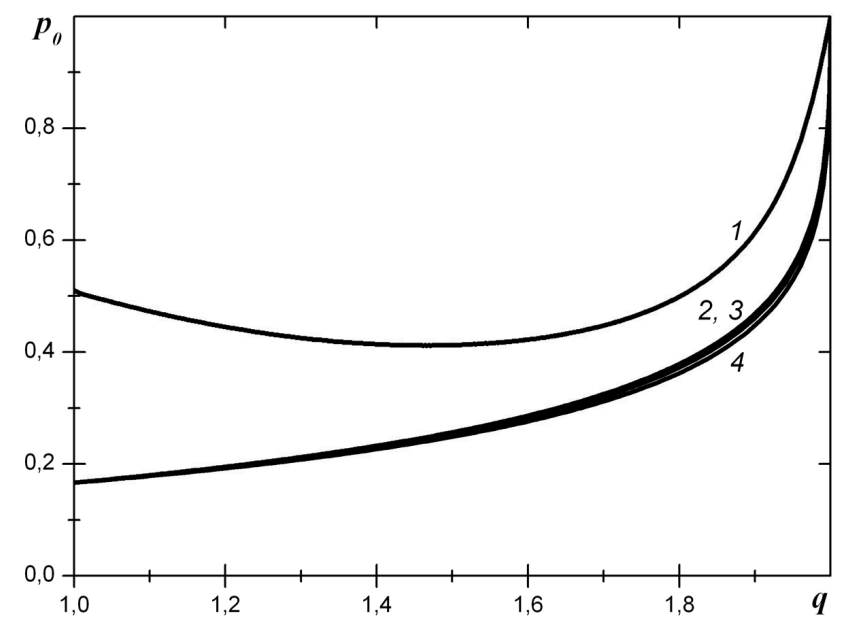

Fig. 7. Top level probabilities for the free-scale, regular, Fibonacci and degenerate trees (curves 1-4, respectively) as function of the deformation parameter at $\Delta=2, b=2$ and $n=5$.

\section{ARBITRARY TREE}

Now, we are in position to consider an arbitrary hierarchical tree, over whose levels $l=0,1, \ldots, n, n \geq 1$ are distributed $N_{l}$ nodes $i_{0} i_{1} \ldots i_{l}$ with the probabilities $p_{i_{0} i_{1} \ldots i_{l}}{ }^{1}$. The main peculiarity of hierarchical trees is known to be a clustered structure, whose fragment is depicted in Fig. 8: nodes $i_{0} \ldots i_{l-1} i_{l}$ of the $l$ th level form a cluster $i_{0} \ldots i_{l-1}$ on the $(l-1)$-level; in turn, clusters $i_{0} \ldots i_{l-2} i_{l-1}$ form supercluster $i_{0} \ldots i_{l-2}$ on the following level $l-2$, et cetera. The above clustering process spreads over the upper levels $l-3, l-4, \ldots$ up to the pair of the top levels $l=1$ and $l=0$ where $N_{i_{0}}$ nodes $i_{1}$ form the superior node $i_{0}$. Along this way, the node probabilities on the hierarchical levels ranged bottomup as follows: $p_{i_{0} \ldots i_{n-1} i_{n}}, p_{i_{0} \ldots i_{n-1}}, \ldots, p_{i_{0} \ldots i_{l}}, \ldots, p_{i_{0} i_{1}}$, $p_{i_{0}} \equiv p_{0}$. Let us calculate these probabilities considering hierarchical levels top-down.

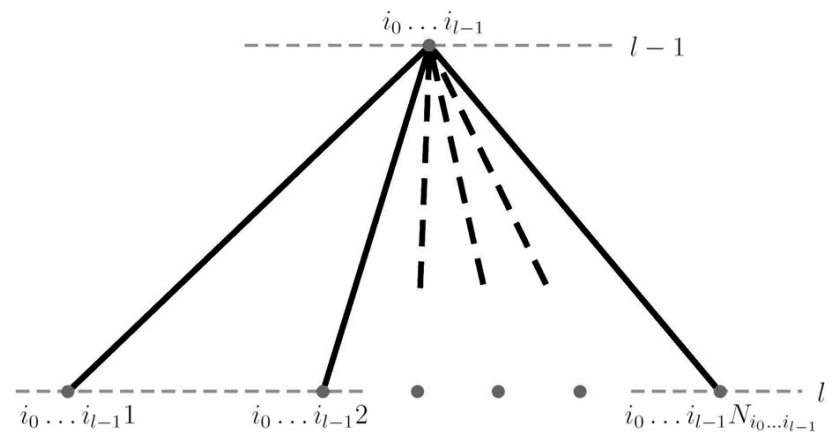

Fig. 8. Node parametrization within a hierarchical cluster.
On the uppermost level $l=0$, one has a single node $i_{0}=1$ related to the probability $p_{0} \equiv p_{i_{0}}$. With the passage down to the level $l=1$, this node multifurcates into a cluster comprising of $N_{i_{0}}$ nodes $i_{1}$. Because of the identity of these nodes, they are characterized by equal probabilities

$$
p_{i_{0} i_{1}}=p_{0} N_{i_{0}}^{-1} \text {. }
$$

In a similar manner, on the following level $l=2$ one obtains the node probabilities

$$
p_{i_{0} i_{1} i_{2}}=p_{i_{0} i_{1}} N_{i_{0} i_{1}}^{-1}=p_{0}\left(N_{i_{0}} N_{i_{0} i_{1}}\right)^{-1} .
$$

The iteration of this procedure down to an arbitrary level $l$ yields the required result

$$
p_{i_{0} \ldots i_{l}}=p_{0}\left(\prod_{m=0}^{l-1} N_{i_{0} \ldots i_{m}}\right)^{-1}
$$

where $N_{i_{0} \ldots i_{m}}$ is the node number within the cluster $i_{0} \ldots i_{m}$.

Generalization of the first equality (25) leads to the expression of the creation probability of an arbitrary level $l$ through a set of related node probabilities. This expression is reduced to the following $l$-fold deformed sum:

$$
\frac{p_{l}}{p_{0}}=\biguplus_{i_{1}=1}^{N_{i_{0}}} \cdots \biguplus_{i_{l}=1}^{N_{i_{0} \ldots i_{l}-1}} \frac{p_{i_{0} \ldots i_{l}}}{p_{0}}, \quad l \neq 0 .
$$

Respectively, the normalization condition (19) takes the form

$$
p_{0} \oplus_{q} p_{0} \biguplus_{i_{1}=1}^{N_{i_{0}}} \cdots \biguplus_{i_{n}=1}^{N_{i_{0} \ldots i_{n-1}}} \frac{p_{i_{0} \ldots i_{n}}}{p_{0}}=1 .
$$

Above, we have used the notation of the deformed sum of $n$ terms:

$$
\biguplus_{i=1}^{n} a_{i} \equiv a_{1} \oplus_{q} a_{2} \oplus_{q} \cdots \oplus_{q} a_{n} .
$$

It is worth noting the characteristic peculiarity of the above consideration: the node probabilities (48) are determined making use of non-deformed algebra, while definition (49) of the level probabilities $p_{l}$ is based on the use of deformed summation (51). A ground of such a partitioning is that the former of these probabilities relates to the configuration of hierarchical trees, while the latter describes their statistical properties.

In conclusion, we consider two examples of the applications of the above theory, among which the former

\footnotetext{
${ }^{1}$ In accordance with Ref. [28], a node coordinate of a hierarchical tree represents so called $p$-adic number $i_{0} i_{1} \ldots i_{n}$ where the first digit $i_{0}=1$ relates to the major ancestor on the uppermost level $l=0$, the second $i_{1}$ numbers are its sons on the lower level $l=1$, and so on - up to the last digit $i_{n}$ numbering the lowest descendants on the bottom level $l=n$.
} 
concerns the Fibonacci tree (Fig. 1(b)), while the latter relates to the schematic evolution tree shown in Fig. 9 (in the last case, nodes identify substantial stages in the evolution of life, e.g., human is situated on the 24th level). Using formulas (48) and (49) for the node and level probabilities, obeying the normalization condition (50), we show that the probability distributions of the Fibonacci tree depicted in Figs. 5-7 do not differ actually from related dependencies for both regular and degenerate trees. Concerning the evolution tree, its probability distributions (Fig. 10) show that the presence of the stopped branches (type of two rightmost ones in Fig. 9) considerably decreases the creation probability of new hierarchical levels. Particularly, the probability of appearance of man takes the values more than $10^{-4}$ only at the deformation parameter $q=1.9999$.

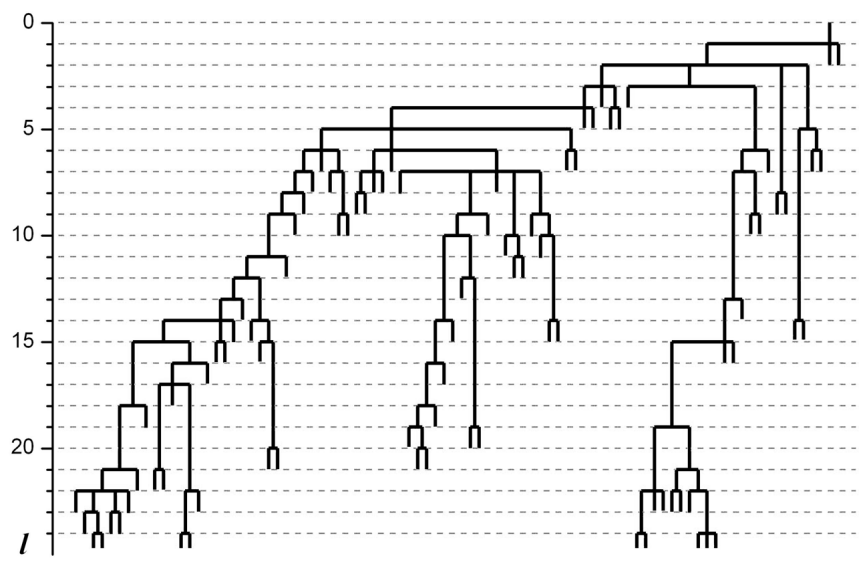

Fig. 9. Schematic representation of evolution tree (from Ref. [42]).

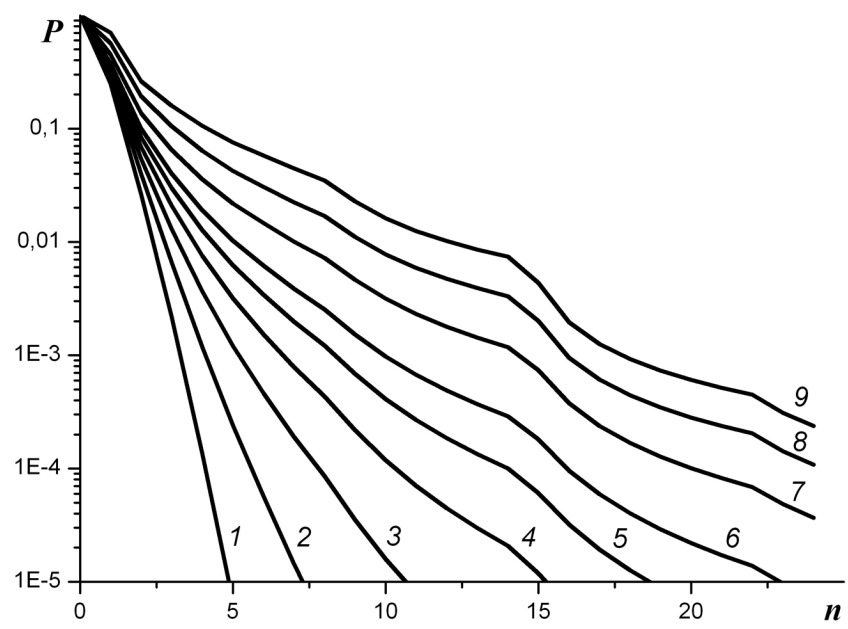

Fig. 10. Creation probability of the evolution tree vs. the level number at: $q=1.0001,1.1,1.2,1.3,1.4,1.5,1.7,1.9$, 1.9999 (curves $1-9$, respectively).

\section{CONCLUDING REMARKS}

To avoid ambiguities it is worthwhile stressing that our consideration concerns rather a probabilistic picture of the creation of the hierarchical trees themselves than hierarchical phenomena and processes evolving on these trees (for example, hierarchically constrained statistical ensembles [13], diffusion processes on multifurcating trees [29], etc.). Among others we have studied analytically both regular and degenerate trees to confirm the coincidence of both analytical and numerical results following from the developed scheme applicable to an arbitrary tree.

A principle peculiarity of the elaborated probabilistic picture is a distinction between the deformed and non-deformed quantities. Thus, effective energies of hierarchical levels in Eq. (20) are non-deformed quantities because the creation of hierarchical structures does not break the conservation law of energy being an additive value. Moreover, the node probabilities are determined making use of non-deformed relations (48) because these probabilities relate to the configuration of the hierarchical tree itself (in other words, they are determined by geometrical, but not probabilistic reasons). At the same time, the hierarchy appearance deforms essentially the probability relations (19), (50), (22), (23) and (24) due to the coupling level probabilities $p_{l}$. Similarly, the definition (49) of these probabilities through the corresponding node values is based on the use of deformed summation (51).

Making use of the deformed algebra leads to an increase of probabilities $p_{l}$ for the whole set of hierarchical levels to take anomalous character near the point $q=2$. The deformed normalization condition (19) is fulfilled only at $q \leq 2$, while it is broken beyond the limit $q=2$. As a result, physically meaningful values of the deformation parameter belong to the domain $q \in[1,2]$.

Our comparison of the probability distributions over hierarchical levels of scale-free, regular, Fibonacci and degenerate trees shows (Fig. 5) that the form of these distributions differs appreciably at all $q$-values only for scale-free tree where the level probability decays much faster. In accordance with such behaviour, the creation probabilities depicted in Fig. 6 decays faster for the scalefree tree than for the other cases. Characteristically, this difference appears within the condition $2-q \ll 1$ only.

Expressions (48)-(50) and (23) constitute the basis for numerical studies of arbitrary hierarchical structures, for example complex defect structures of solids subject to intensive external influence, e.g. a rigid radiation treatment. Unlike in amorphous systems, the number of structure levels of a real crystal is rather not large: usually, among different spatial scales, it is accepted to distinguish micro-, meso- and macroscopic levels [43]. To study a real structure, one needs first to distribute the whole ensemble of defects over hierarchical levels $l=0,1, \ldots, n$; then, one calculates on each of them a number of defects $N_{i_{0} i_{1} \ldots i_{l-1}}$ belonging to the cluster $i_{0} i_{1} \ldots i_{l-1} 1$, $i_{0} i_{1} \ldots i_{l-1} 2, \ldots, i_{0} i_{1} \ldots i_{l-1} N_{i_{1} i_{2} \ldots i_{l-1}}$ and attributes the probability $p_{i_{0} \ldots i_{l}}$ to this cluster in accordance with 
Eq. (48). Next, the level probabilities $p_{l}$ are calculated according to definition (49) where the top value $p_{0}$ is fixed by the normalization condition (50). Finally, the creation probabilities $P_{n}$ of hierarchical trees are determined by equality (23).

\section{APPENDIX A: MAIN RULES OF DEFORMED ALGEBRA}

Following [32], let us present the main equations of the deformed algebra. The related formalism is known to be based on a generalized definition of the logarithm and exponential functions

$$
\begin{array}{r}
\ln _{q}(x):=\frac{x^{1-q}-1}{1-q}, \\
\exp _{q}(x):=[1+(1-q) x]_{+}^{\frac{1}{1-q}}
\end{array}
$$

being characterized by the deformation parameter $q \geq 0$ with the notion $[y]_{+} \equiv \max (0, y)$. For any numbers $x, y>0$, the deformed product and ratio are defined by the following relations:

$$
\begin{aligned}
& x \otimes_{q} y:=\left[x^{1-q}+y^{1-q}-1\right]_{+}^{\frac{1}{1-q}}, \\
& x \oslash_{q} y:=\left[x^{1-q}-y^{1-q}+1\right]_{+}^{\frac{1}{1-q}} .
\end{aligned}
$$

Respectively, the deformed sum and difference read

$$
\begin{aligned}
x \oplus_{q} y & :=x+y+(1-q) x y, \\
x \ominus_{q} y & :=\frac{x-y}{1+(1-q) y}
\end{aligned}
$$

where the condition $y \neq-\frac{1}{1-q}$ is implied. The $n$-fold deformed sum of identical terms is defined as follows:

$$
n \odot_{q} x \equiv \underbrace{x \oplus_{q} x \oplus_{q} \cdots \oplus_{q} x}_{n}:=\frac{[1+(1-q) x]_{+}^{n}-1}{1-q} .
$$

The rules (A.2), (A.3) ensure the following properties of the $q$-logarithm and the $q$-exponential (A.1):

$$
\begin{aligned}
\ln _{q}\left(x \otimes_{q} y\right) & =\ln _{q} x+\ln _{q} y, \\
\ln _{q}\left(x \oslash_{q} y\right) & =\ln _{q} x-\ln _{q} y ; \\
\exp _{q}(x) \otimes_{q} \exp _{q}(y) & =\exp _{q}(x+y), \\
\exp _{q}(x) \oslash_{q} \exp _{q}(y) & =\exp _{q}(x-y) .
\end{aligned}
$$

\section{APPENDIX B: DEFORMED SUM OF TERMS OF A GEOMETRICAL PROGRESSION}

Let a geometrical sequence $a, a r, a r^{2}, \ldots, a r^{n-1}$ be determined by the common ratio $r$, the scale factor $a$ and the term number $n$. With the deformed summation rule (A.3), direct calculations at lower numbers $n=2,3, \ldots$. show the sum of terms of a geometrical progression

$$
S_{n}:=\underbrace{a \oplus_{q} a r \oplus_{q} \oplus_{q} a r^{2} \oplus_{q} \cdots \oplus_{q} a r^{n-1}}_{n}
$$

can be written as the series

$$
S_{n}:=a \sum_{m=0}^{n-1} \sigma_{n}^{m}[(1-q) a]^{m}
$$

with unknown coefficients $\sigma_{n}^{m}$. The iteration of Eq. (B.1) yields the chain of the following relations:

$$
\begin{aligned}
S_{n+1}: & =S_{n} \oplus_{q}\left(a r^{n}\right)=\left(S_{n}+a r^{n}\right)+(1-q) S_{n}\left(a r^{n}\right) \\
& =\left\{a\left(\sigma_{n}^{0}+r^{n}\right)+a \sum_{m=1}^{n-1} \sigma_{n}^{m}[(1-q) a]^{m}\right\} \\
& +(1-q) a\left(a r^{n}\right) \sum_{m=0}^{n-1} \sigma_{n}^{m}[(1-q) a]^{m} \\
& =a \sigma_{n+1}^{0}+a \sum_{l=0}^{n-2} \sigma_{n}^{l+1}[(1-q) a]^{l+1} \\
& +a[(1-q) a] r^{n} \sum_{m=0}^{n-1} \sigma_{n}^{m}[(1-q) a]^{m} \\
& =a \sigma_{n+1}^{0}+a \sum_{l=0}^{n-2}\left(\sigma_{n}^{l+1}+r^{n} \sigma_{n}^{l}\right)[(1-q) a]^{l+1} \\
& +a[(1-q) a] r^{n} \sigma_{n}^{n-1}[(1-q) a]^{n-1} \\
& =a \sigma_{n+1}^{0}+a \sum_{m=1}^{n-1}\left(\sigma_{n}^{m}+r^{n} \sigma_{n}^{m-1}\right)[(1-q) a]^{m} \\
& +a \sigma_{n}^{n-1}[(1-q) a]^{n} r^{n} .
\end{aligned}
$$

Here, the first line takes into account definition (A.3); in the second line, series (B.2) is applied to single out the term related to $m=0$ within the braces; in the fourth line, the first term is written in accordance with definition (B.2) related to the term $m=0$, while the summation index $l=m-1$ is introduced in the second term; in the sixth line, the second term contains both sums over $l$ and $m$ of the previous line, the last term relates to the index $m=n-1$; in the eighth line, we return to the summation index $m=l+1$. As a result, series (B.2) takes the form

$$
\begin{array}{r}
S_{n}=a s_{n}+a \sum_{m=1}^{n-2}\left(\sigma_{n-1}^{m}+r^{n-1} \sigma_{n-1}^{m-1}\right)[(1-q) a]^{m} \\
+a \sigma_{n-1}^{n-2}[(1-q) a]^{n-1} r^{n-1}
\end{array}
$$

where the sum

$$
s_{n} \equiv \sum_{m=0}^{n-1} r^{m}=\frac{1-r^{n}}{1-r}
$$

of the ordinary geometrical progression $1, r, r^{2}, \ldots, r^{n-1}$ was used. The comparison of the terms of Eqs. (B.2) and (B.4) related to the equal $m$ indices arrives at the following recursion relations: 


$$
\begin{aligned}
& \sigma_{n}^{0}=s_{n} ; \\
& \sigma_{n}^{m}=\sigma_{n-1}^{m}+\sigma_{n-1}^{m-1} r^{n-1}, \quad m \in[1, n-2] \\
& \sigma_{n}^{n-1}=\sigma_{n-1}^{n-2} r^{n-1} .
\end{aligned}
$$

The first of these terms gives an explicit expression of the lowest power coefficient in series (B.2). It is easy to convince oneself that regression (B.7) is satisfied with the insertion

$$
\sigma_{n}^{m}=\sum_{l=0}^{n-1} \sigma_{l}^{m-1} r^{l}
$$

whose iteration yields

$$
\begin{aligned}
& \sigma_{n}^{m}=\sum_{l=0}^{n-1} \sigma_{l}^{m-1} r^{l}=\sum_{l=0}^{n-1} \sum_{k=0}^{l-1} \sigma_{k}^{m-2} r^{l+k}=\ldots \\
& =\sum_{l_{m-1}=0}^{n-1} r^{l_{m-1}} \sum_{l_{m-2}=0}^{l_{m-1}-1} r^{l_{m-2}} \cdots \sum_{l_{0}=0}^{l_{1}-1} \sigma_{l_{0}}^{0} r^{l_{0}} .
\end{aligned}
$$

However, the last expression is inconvenient for direct calculations because it contains connected exponents of the ratio $r$ with the upper limits of the consequent sums. Hence, let us calculate explicitly the coefficients (B.9) for small indices $m$ :

$$
\begin{aligned}
\sigma_{n}^{1} & =\sum_{l=0}^{n-1} \sigma_{l}^{0} r^{l}=\sum_{l=0}^{n-1} s_{l} r^{l}=\sum_{l=0}^{n-1} \frac{1-r^{n}}{1-r} r^{l} \\
& =r \frac{\left(1-r^{n}\right)\left(1-r^{n-1}\right)}{(1-r)\left(1-r^{2}\right)}, \\
\sigma_{n}^{2} & =\sum_{l=0}^{n-1} \sigma_{l}^{1} r^{l}=r \sum_{l=0}^{n-1} \frac{\left(1-r^{l}\right)\left(1-r^{l-1}\right)}{(1-r)\left(1-r^{2}\right)} r^{l} \\
& =r^{3} \frac{\left(1-r^{n}\right)\left(1-r^{n-1}\right)\left(1-r^{n-2}\right)}{(1-r)\left(1-r^{2}\right)\left(1-r^{3}\right)},
\end{aligned}
$$

These expressions show that the above coefficients are proportional to the fractions, whose denominators represent the production of the terms $1-r^{l+1}$ with growing powers $l=0,1, \ldots$, while the numerators contain the same number of the terms $1-r^{n-l}$ with decreasing powers. As a result, we suppose the coefficients to be found in the following form:

$$
\begin{aligned}
\sigma_{n}^{m} & =r^{\sum_{k=1}^{m} k} \prod_{l=0}^{m} \frac{1-r^{n-l}}{1-r^{l+1}} \\
& =r^{\frac{m(m+1)}{2}} \prod_{l=0}^{m} \frac{1-r^{n-l}}{1-r^{l+1}} .
\end{aligned}
$$

At $m=0$ this equality is reduced to the condition (B.6). Respectively, at $m \in[1, n-2]$ inserting (B.12) into (B.7) arrives at the following relations:

$$
\begin{aligned}
\sigma_{n}^{m} & =r^{\sum_{k=1}^{m} k} \prod_{l=0}^{m} \frac{1-r^{n-1-l}}{1-r^{l+1}}+r^{\sum_{k=1}^{m-1} k} \prod_{l=0}^{m-1} \frac{1-r^{n-1-l}}{1-r^{l+1}} r^{n-1} \\
& =r^{\sum_{k=1}^{m} k} \prod_{l=0}^{m} \frac{1-r^{n-1-l}}{1-r^{l+1}}\left(1+\frac{1-r^{m+1}}{1-r^{n-m-1}} r^{n-1-m}\right)=r^{\sum_{k=1}^{m} k} \frac{\prod_{l=1}^{m+1}\left(1-r^{n-l}\right)}{\prod_{l=0}^{m}\left(1-r^{l+1}\right)} \frac{1-r^{n}}{1-r^{n-m-1}} \\
& =r^{\sum_{k=1}^{m} k} \frac{\prod_{l=0}^{m}\left(1-r^{n-l}\right) \frac{1-r^{n-(m+1)}}{1-r^{n}}}{\prod_{l=0}^{m}\left(1-r^{l+1}\right)} \frac{1-r^{n}}{1-r^{n-m-1}}=r^{\frac{m(m+1)}{2}} \prod_{l=0}^{m} \frac{1-r^{n-l}}{1-r^{l+1}} .
\end{aligned}
$$

In the third line, the overall multiplier is singled out from terms of the second line; the last fraction in the fourth line is obtained with obvious summation in brackets of the previous line; the fraction in the numerator of the first fraction in the fifth line appears to single out the multipliers related to both lower $l=0$ and upper $l=m+1$ limits in the upper production; the last line is the result of reduction of fractions in the previous line. Finally, at $m=n-1$ Eqs. (B.8) and (B.12) take the equal form

$$
\sigma_{n}^{n-1}=r^{\frac{n(n-1)}{2}} .
$$

Thus, one can conclude that proposition (B.12) is applicable for all indices $m \in[0, n-1]$ and its insertion into
Eq. (B.2) arrives at the final expression of the sum of the terms of a geometrical progression (B.1):

$$
S_{n}=a \sum_{m=0}^{n-1} C_{n}^{m+1}(r) r^{\frac{m(m+1)}{2}}[(1-q) a]^{m}
$$

with the coefficients

$$
C_{n}^{m}(r) \equiv \prod_{l=0}^{m-1} \frac{1-r^{n-l}}{1-r^{l+1}}
$$

Expression (B.15) can be written within the product representation according to the relations 


$$
\begin{array}{r}
S_{n}=\frac{1}{1-q}\left\{\sum_{m=0}^{n} C_{n}^{m}(r) r^{\frac{m(m-1)}{2}}[(1-q) a]^{m}-1\right\} \\
=\frac{1}{1-q}\left\{\prod_{m=0}^{n-1}\left[1+a(1-q) r^{m}\right]-1\right\},
\end{array}
$$

the second of which expresses the deformed Gauss polynomials $[33,44]$

$$
\begin{array}{r}
\sum_{m=0}^{n} C_{n}^{m}(r) r^{\frac{m(m-1)}{2}}[(1-q) a]^{m} \\
=\prod_{m=0}^{n-1}\left[1+a(1-q) r^{m}\right] .
\end{array}
$$

Taking into account definition (A.4), one obtains at $r=1$

$$
S_{n}=\frac{[1+(1-q) a]^{n}-1}{1-q}=n \odot_{q} a .
$$

In the non-deformed limit $q \rightarrow 1$, this relation takes the trivial form $S_{n}=n a$.

Rewriting definition (B.16) in the forms

$$
\begin{gathered}
C_{n}^{m}(r)=\frac{\prod_{l=(n-m)+1}^{n}\left(1-r^{l}\right)}{\prod_{l=1}^{m}\left(1-r^{l}\right)} \\
=\frac{\prod_{l=1}^{n}\left(1-r^{l}\right)}{\prod_{l=1}^{m}\left(1-r^{l}\right) \prod_{l=1}^{n-m}\left(1-r^{l}\right)},
\end{gathered}
$$

one can see that coefficients (B.16) are reduced to the deformed binomial coefficients $[33,44]$

$$
C_{n}^{m}(r) \equiv \frac{[n]_{r} !}{[m]_{r} ![n-m]_{r} !}
$$

determined with the deformed factorial

$$
\begin{aligned}
& {[n]_{r} ! \equiv[1]_{r}[2]_{r} \ldots[n]_{r}} \\
& =\frac{(r-1)\left(r^{2}-1\right) \ldots\left(r^{n}-1\right)}{(r-1)^{n}} \\
& {[n]_{r} \equiv 1+r+r^{2}+\cdots+r^{n-1}=\frac{r^{n}-1}{r-1}}
\end{aligned}
$$

According to formula (B.21), the deformed binomial coefficients obey the usual property

$$
C_{n}^{m}(r)=C_{n}^{n-m}(r)
$$

After replacing index $m+1$ by $m$ in Eq. (B.7) taking into account Eq. (B.12), we arrive at the deformed Pascal identity

$$
C_{n}^{m}(r)=C_{n-1}^{m}(r)+C_{n-1}^{m-1}(r) r^{n-m}
$$

that forms the deformed Pascal triangle

$$
\begin{aligned}
& 1
\end{aligned}
$$

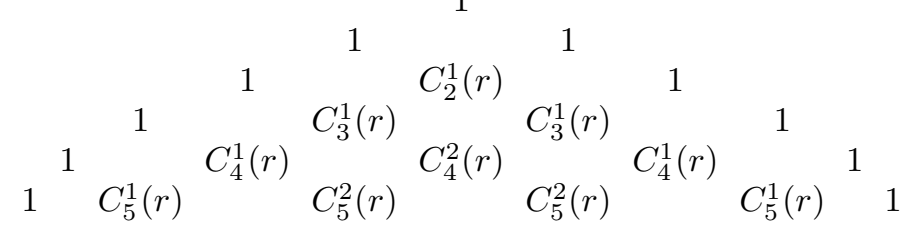

where we put $C_{n}^{0}(r)=1$. On the other hand, the iteration of relation (B.7) yields the sum rule

$$
C_{n}^{m}(r)=C_{n-1}^{m}(r)+\left[C_{n-2}^{m-1}(r)+C_{n-2}^{m-2}(r) r^{n-m}\right] r^{n-m}=\cdots=\sum_{l=0}^{m} C_{n-(l+1)}^{m-l}(r) r^{(n-m) l} .
$$

Finally, in limit $r \rightarrow 1$ relation (B.21) takes an ordinary form:

$$
\lim _{r \rightarrow 1} C_{n}^{m}(r)=\lim _{r \rightarrow 1} \prod_{l=0}^{m} \frac{1-r^{n-l}}{1-r^{l+1}}=\prod_{l=0}^{m-1} \frac{n-l}{l+1} \equiv \frac{n !}{m !(n-m) !} .
$$

\section{APPENDIX C: OBTAINING ASYMPTOTIC FORMS OF DISTRIBUTIONS (26) AND (42)}

The simplest way to find the $b \rightarrow \infty$ limit of the binomial in distribution (26) is to take its logarithm and then use the expansion over powers of $b^{-l}$ :

$$
\begin{aligned}
& \ln \left[1+(1-q) b^{-l}\right]_{+}^{b^{l}}=b^{l} \ln \left[1+(1-q) b^{-l}\right]_{+} \\
& \simeq b^{l}\left[(1-q) b^{-l}-\frac{1}{2}(1-q)^{2} b^{-2 l}\right] \\
& =(1-q)\left(1+\frac{q-1}{2} b^{-l}\right) .
\end{aligned}
$$


The potentiation of the last expression and the insertion of the obtained result into Eq. (26) yields the asymptotics (29).

According to definition (43) the basic-deformed binomial in Eq. (42) takes the explicit form

$$
\begin{aligned}
& \left(1+\frac{1-q}{b}\right)_{1 / b}^{(b-1) l}=\left(1+\frac{1-q}{b}\right)^{b-1}\left(1+\frac{1-q}{b^{2}}\right)^{b-1} \\
& \ldots\left(1+\frac{1-q}{b^{l}}\right)^{b-1} .
\end{aligned}
$$

In the limit $b \rightarrow \infty$, the first factor takes the value $\mathrm{e}^{1-q}$, while the rest of production is estimated as

$$
1+(q-1) b^{-l}+\frac{(q-1)^{2}}{2} b^{-2 l} .
$$

In order to obtain this estimation, firstly, each of the factors in (C.2) has been expanded into a series up to the square term, then the brakets have been removed and, finally, all the factors before each power of $1-q$ have been collected and reduced. Inserting the obtained estimation into the probability distribution (42) and taking into account the terms $1-q$ up to the second order leads to the asymptotic form (44) of this distribution.
[1] L. Whyte, A. G. Wilson, D. Wilson (Eds.), Hierarchical structures, hierarchical thinking (American Elsevier, New York, 1969).

[2] H. H. Pattee (Eds.), Hierarchy theory: the challenge or complex systems (Braziller, New York, 1973).

[3] T. F. H. Allen, T. B. Starr, Hierarchy: perspectives for ecological complexity (University Chicago Press, Chicago, 1982).

[4] S. Salthe, Evolving Hierarchical Systems: their structure and representation (Columbia U.P., New York, 1985).

[5] J. S. Nicolis, Dynamics of Hierarchical Systems, (An Evolutionary Approach) (Springer-Verlag, Berlin, Heidelberg, 1986).

[6] S. Kauffman, The Origins of Order (Oxford U.P., Oxford, 1993).

[7] G. J. Dalenoort, The Paradigm of self-Organization II (Gordon \& Breach, London, 1994).

[8] J. Holland, Hidden Order (Addison Wesley, New York, 1995)

[9] A. I. Olemskoi, in Fractals in Condensed Matter Physics, edited by I. M. Khalatnikov, Phys. Rev. 18, Part 1 (Gordon \& Breach, London, 1996).

[10] K. Mainzer, Thinking in Complexity (Springer-Verlag, Berlin, 1997).

[11] R. N. Mantegna, H. E. Stanley, An introduction to econophysics. Correlations and complexity in finance (Cambridge University Press, Cambridge, 1999).

[12] P. Holme, M. Huss, H. Jeong, Bioinformatics 19, 532 (2003).

[13] A. I. Olemskoi, V. I. Ostrik, S. V. Kokhan, Physica A 388, 609 (2009).

[14] E. Ravasz, A.-L. Barabási, Phys. Rev. E 67, 026112 (2003).

[15] Yu. Holovatch et al., J. Phys. Stud. 10, 247 (2006).

[16] R. Albert, H. Jeong, A.-L. Barabási, Nature 401, 130 (1999).

[17] H. Jeong, B. Tombor, R. Albert, Z. Oltvai, A.-L. Barabási, Nature 407, 651 (2000).

[18] A. Wagner, D. A. Fell, Proc. R. Soc. Lond. B. Biol. Sci. 268, 1803 (2001).

[19] H. Jeong, S. Mason, A.-L. Barabási, Z. N. Oltvai, Nature 411, 41 (2001).

[20] A. Wagner, Mol. Biol. Evol. 18, 1283 (2001).

[21] M. E. J. Newman, Proc. Nat. Acad. Sci. U.S.A. 98, 404
(2001)

[22] M. E. J. Newman, Phys. Rev. E 64, 016131 (2001).

[23] A.-L. Barabási, H. Jeong, Z. Néda, E. Ravasz, A. Schubert, T. Vicsek, Physica A 311, 590 (2002).

[24] A.-L. Barabási, R. Albert, Science 286, 509 (1999).

[25] A.-L. Barabási, R. Albert, H. Jeong, Physica A 272, 173 (1999).

[26] M. S. Granovetter, Am. J. Sociol. 78, 1360 (1973).

[27] G. W. Flake, S. Lawrence, C. L. Giles, in Proceedings of the Sixth International Conference on Knowledge Discovery and Data Mining, ACM, Boston, MA, USA, 2000, p. 150 .

[28] R. Rammal, G. Toulouse, M. A. Virasoro, Rev. Mod. Phys. 58, 765 (1986).

[29] C. P. Bachas, B. A. Huberman, Phys. Rev. Lett. 57, 1965 (1986)

[30] A. I. Olemskoi, JETP Lett. 71, 285 (2000).

[31] C. Tsallis, Introduction to Nonextensive Statistical Mechanics - Approaching a Complex World (Springer, New York, 2009).

[32] E. P. Borges, Physica A 340, 95 (2004).

[33] V. Kac, P. Cheung, Quantum calculus (Springer, New York, 2002).

[34] B. Corominas-Murtra, J. Goñi, C. Rodríguez-Caso, R. Solé, arXiv:1011.4394.

[35] M. E. J. Newman, SIAM Review 45, 167 (2003).

[36] K. G. Wilson, J. Kogut, Phys. Rep. 12, 75 (1974).

[37] A. I. Olemskoi, D. O. Kharchenko, J. Phys. Stud. 6, 253 (2002).

[38] A. I. Olemskoi, A. S. Vaylenko, I. A. Shuda, Physica A 388, 1929 (2009).

[39] C. Tsallis, R. S. Mendes, A. R. Plastino, Physica A 261, 534 (1998).

[40] A. I. Olemskoi, I. A. Shuda, V. N. Borisyuk, Eur. Phys. Lett. 89, 50007 (2010).

[41] A. I. Olemskoi, A. D. Kiselev, Phys. Lett. A 247, 221 (1998).

[42] A. M. Sugden, B. R. Jasny, E. Culotta, E. Pennisi, Science 300, 1691 (2003).

[43] A. I. Olemskoi, Theory of Structure Transformations in Non-Equilibrium Condensed Matter (NOVA Science, New York, 1999).

[44] H. Exton, q-Hypergeometric functions and applications (Ellis Horwood, Chichester, 1983). 


\section{ІМОВІРНОСТІ УТВОРЕННЯ ІЕРАРХІЧНИХ ДЕРЕВ}

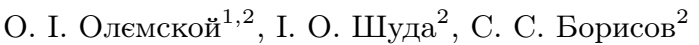

${ }^{1}$ Інститут прикладної фізики НАН Украйни, вул. Петропавлівсъка, 58, 400зо, м. Суми, Украӥна

${ }^{2}$ Сумсъкий державний університет, вул. Римсъкого-Корсакова, 2, 40007, м. Суми, Україна

Ми розглядаємо аналітично й чисельно умови утворення різних ієрархічних дерев. Досліджуємо зв'язок між імовірностями утворення ієрархічних рівнів та ймовірності об'єднання цих рівнів у єдину структуру. Показуємо, що побудова послідовної ймовірнісної картини вимагає використання деформованої алгебри. Наш розгляд заснований на вивченні основних типів ієрархічних дерев, серед яких регулярне й вироджене досліджені аналітично, тоді як імовірності утворення дерева Фібоначчі та самоподібного дерева визначені чисельно. Ми знаходимо загальний вираз для ймовірності утворення довільного дерева й обчислюємо суму членів деформованої геометричної прогресії, яка з'являється при розгляді виродженого дерева. 